Ann. Biol. anim. Bioch. Biophys., I969, 9 (2), 205-218.

\title{
ÉTUDE DES LACTICODÉSHYDROGÉNASES PLASMATIQUE ET HÉPATIQUE CHEZ L'OIE AU COURS D'Une STÉtTose PROVOQUÉE PAR GAVAGE
}

\author{
Odette IVOREC-SZYLIT * et M. SZYLIT ** \\ avec la collaboration technique de Mme FENDRY \\ et de MM. Rousselot, Morin et Monachon \\ * Station de Recherches Avicoles, \\ Centre national de Recherches zootechniques, 78 -Jouy-en-Josas \\ Institut national de la Recherche agronomique \\ ** Laboratoire de Biologie Physico-chimique, \\ Faculté des Sciences, 91 - Orsay
}

\section{SOMMAIRE}

On étudie l'évolution chez l'Oie, des lacticodéshydrogénases hépatique (F-LDH) et plasmatique (S-LDH) au cours d'une stéatose provoquée par 3 semaines de gavage et après l'interruption de ce dernier à différents stades.

L'analyse quantitative (activité totale) et qualitative (proportion relative des iso-enzymes) montre que l'excès d'activité de la S-LDH provient principalement de la LDH du foie. Les expériences d'interruption de gavage confirment cette relation et suggèrent une évolution des mécanismes de libération de la F-LDH au cours du gavage.

L'évolution de la S-LDH est discontinue : accroissement rapide les 8 premiers jours, régression puis stabilisation ou légère augmentation jusqu'au $2 \mathrm{I}^{\mathrm{e}}$ et dernier jour de gavage.

L'accroissement de la perméabilité cellulaire de l'hépatocyte, mesuré par l'élévation du taux de la LDH plasmatique semble être la première réponse au gavage, biẹn avant l'apparition de toutes manifestations histologiques. En fin de gavage, l'activité S-LDH serait une mesure de l'altération cellulaire.

\section{INTRODUCTION}

La stéatose hépatique de 1'Oie provoquée par une alimentation forcée est caractérisée par une perturbation importante du métabolisme des lipides et des hydrates de carbone (FORENBACHER, I967). Nous avons peu de renseignements sur l'évolution des enzymes de la cellule hépatique. Cependant, des expériences préliminaires (SzyLIT 
et IVOREC-SzYLIT, I967 ; SzYLIT, LECLERC et IVOREC-SzYLIT, I968) ont montré en fin de gavage un accroissement important de l'activité des lacticodéshydrogénases du plasma et du tissu hépatique.

L'étude de cet enzyme est particulièrement intéressante car la $\mathrm{L}$, DH joue un rôle essentiel dans 1'utilisation des réserves énergétiques par la voie glycolytique et intervient dans les processus gluconéogéniques. Cet enzyme existe sous 5 formes moléculaires différentes (iso-enzymes) (MARKERT et MOLLER, I959) en proportions variables suivant les tissus considérés. Ces proportions dans le sérum sont relativement stables quand l'activité métabolique des différents tissus de l'organisme est normale. Lorsqu'il y a une altération de l'intégrité cellulaire ou de l'activité synthétique d'un organe, la concentration de la S-LDH est accrue et sa composition, modifiée de façon spécifique, tend à se rapprocher de celle de l'enzyme de cet organe. Ceci, en particulier, est vérifié chez l'Homme dans certaines manifestations pathologiques du tractus hépatobiliaire (WROBLEWSKI et GREGORY, I96r). L'étude quantitative et qualitative de la S-I $\mathrm{LDH}$ permet donc de préciser l'organe lésé et l'importance du dommage.

C'est dans cet esprit que nous avons suivi l'évolution des LDH hépatique et plasmatique, d'une part au cours d'une stéatose provoquée par gavage, d'autre part après interruption de cette dernière à différents stades.

\section{MATÉRIELS ET MÉ'THODES}

\section{Animaux et conditions de nutrition}

L'expérience est poursuivie sur 29 oies de race Landaise, âgées de 7 mois, réparties en 3 lots $\mathrm{A}, \mathrm{B}$ et $\mathrm{C}$. Les animaux des deux premiers groupes servent de témoins. Le lot A groupe 4 animaux nourris ad libitum avec un aliment composé à base de céréales et d'herbe fraîche. Les autres animaux sont prégavés pendant dix jours avec un régime enrichi de $50 \mathrm{p}$. Ioo de farine de viande, puis reçoivent l'aliment de gavage (maïs bouilli). Les 4 animaux du lot B sont nourris ad libitum; les $2 \mathrm{I}$ animaux du lot $\mathrm{C}$ sont gavés à raison de I $300 \mathrm{~g}$ d'aliment en moyenne par animal et par jour, distribués en 3 fois. Ce dernier lot est subdivisé en 3 groupes :

- Sept oies sont gavées normalement pendant $2 \mathrm{I}$ jours.

- Sept sont abattues en cours de gavage au bout de 4 et Ir jours.

- enfin, le gavage du dernier groupe (oies "dégavées ") est interrompu après 7,14 et 21 jours et le même régime est distribué ad libitum.

\section{Prélèvements et préparations des échantillons}

Le sang est recueilli sur anticoagulant (citrate de sodium à $3,8 \mathrm{p}$. 100) par des prises de $5 \mathrm{ml}$ à la veine alaire. Ces prélèvements ont lieu deux fois par semaine entre 8 et II heures. Les plasmas sont conservés à $4^{\circ} \mathrm{C}$ après avoir écarté les échantillons hémolysés.

Les animaux sont sacrifiés à jeun par saignée. Le foie et le pancréas sont immédiatement prélevés et congelés. Les échantillons analysés de foie proviennent tous du milieu du petit lobe. Le foie est broyé (broyeur Ultra-Turrax) à raison de $16 \mathrm{ml}$ de sérum physiologique refroidi par gramme de matière fraîche et centrifugé $20 \mathrm{mn}$ à $140000 \mathrm{~g}$. Les lipides qui se regroupent à la surface sont éliminés. Le culot est broyé à nouveau 2 fois puis rejeté après une heure de centrifugation. Les 3 surnageants sont réunis et on mesure immédiatement l'activité de la $\mathrm{LDH}$, la concentration en protéines solubles et en acide lactique.

\section{Dosages}

Le dosage spectrophotométrique de la LDH suivant la technique de KuBowitz-OTT (1943) a été décrit par ailleurs (Szylit et IvOREc-SzYlit, I967) ; on suit la vitesse d'oxydation de NADH en 
$\mathrm{NAD}^{+} \mathrm{à} 340 \mathrm{~m} \mu$, ̀̀ $\mathrm{pH} 7,6$ et à $25^{\circ} \mathrm{C}$. La cinétique est d'ordre o pendant les 3 premières minutes. On vérifie que l'activité mesurée est bien proportionnelle au volume de l'échantillon : dans le cas du sérum, celui-ci est compris entre 5 et 50 microlitres suivant que l'animal est gavé ou non. Les homogénats de foie sont dilués dans une solution tampon phosphate $0, \mathrm{I} \mathrm{M}$ à $\mathrm{pH} ~ 7,6$ de façon à obtenir une variation de densité optique de l'ordre de 0,05 par minute, pour un volume total de $2,3 \mathrm{ml}$. En absence de pyruvate exogène, il n'y a pas d'oxydation parasite de NADH.

L'activité est exprimée en unités internationales : quantité de LDH qui réduit une micromole de pyruvate par minute, dans les conditions de dosage. Les résultats sont exprimés en unités internationales par $\mu \mathrm{l}$ de plasma et par foie entier. Les protéines solubles sont estimées par la méthode de WARBURg et Christian (194I). Le dosage de l'acide iactique a déjà été décrit (Ivorec-SzyLiT et SzYLIT, I965).

Les ultracentrifugations analytiques sont effectuées à $20^{\circ} \mathrm{C}$ en milieu chlorure de sodium o,I54 M pour les extraits de foie et en milieu tampon phosphate 7,6 pour les sérums. L'électrophorèse sur gel d'acrylamide est conduite suivant une microméthode mise au point par l'un d'entre nous (SzYLIT, 1968). La migration s'effectue pendant $40 \mathrm{mn}$ à $7^{\circ} \mathrm{C}$ dans un tampon Tris-EDTA-acide borique à $\mathrm{pH}$ 9, $\mathrm{r}$ sous une différence de potentiel de 300 volts. Les protéines sont colorées par le Bleu de Comassie suivant Chrambach (1967), et les zones à activité LDH sont repérées in situ par une suite de réactions d'oxydo-réductions spécifiques de cet enzyme (DEWEY et ConkLin, I960). Le terme final de ces réactions est un composé insoluble et fortement coloré.

\section{RÉSULTATS}

\section{Résultats zootechniques}

La consommation est contrôlée chaque jour pour les trois lots d'animaux. Les résultats sont exprimés sur la figure $\mathrm{I} a$. Dans le cas des animaux témoins des lots $\mathrm{A}$ et $B$, les variations de la consommation moyenne sont quelconques. Le déroulement du processus alimentaire du gavage est le suivant : la valeur de la ration quotidienne croît de $600 \mathrm{~g}$ à $\mathrm{I} 500 \mathrm{~g}$ pendant la première moitié de l'expérience, reste constante puis diminue quelque peu pendant les derniers jours.
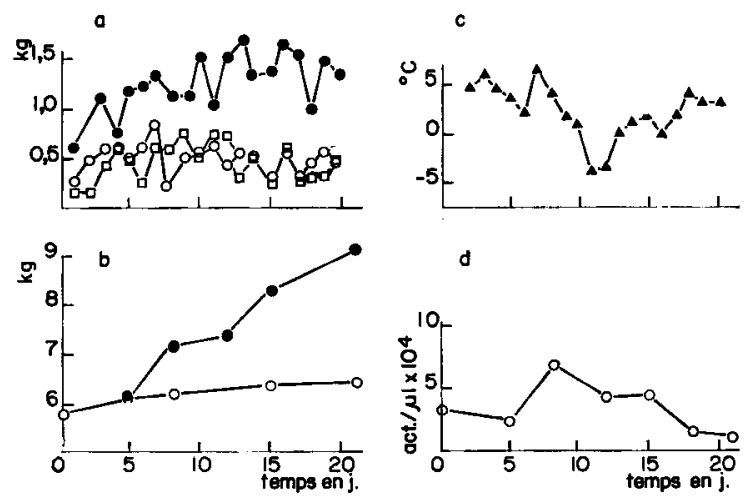

$d$

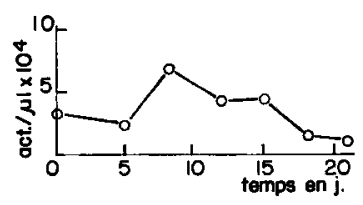

FIG. I. - Evolution de la consommation moyenne par animal et par jour (I a), $d u$ poids corporel ( $\mathrm{I} b$ ), de la température ambiante moyenne ( $\mathrm{c} c$ ) et de la $S$-LDH (I d) pendant les 21 jours qui suivent le début du gavage (o) et (a) : témoins; gavés $(\bullet)$

Le gain de poids a également été contrôlé au cours de l'expérience. Les résultats groupés sur la figure $\mathrm{I} b$, montrent que le poids des animaux témoins augmente de Io p. roo, celui des oies gavées de $50 \mathrm{p}$. Ioo. Les foies gavés obtenus étaient dans l'ensemble de bonne qualité. 


\section{Vérification de la stabilité de la $S-L D H$ et de la $F-L D H$}

Tous les sérums composant une série sont analysés le même jour mais les différentes séries sont étudiées à des intervalles de temps variables ( 2 à 5 jours) par rapport à la date du prélèvement. De même, certains foies sont conservés entiers à l'état congelé plus longtemps que d'autres. Les conséquences possibles de ces décalages sur la mesure de 1'activité ont été analysées. Les résultats qui figurent sur le tableau I montrent qu'elles sont nulles dans nos conditions de travail. Les lacticodéshydrogénases du plasma et du foie de l'Oie comme celles des autres espèces apparaissent comme des enzymes très stables (SHoNk et BoxkR, I964).

\section{TABLEAU I}

Étude de la stabilité de la $L D H$ du plasma et du foie Les activités sont mesurées sur le même échantillon

\begin{tabular}{|c|c|c|c|}
\hline S. LDH & Activité (\%) & F. LDH & Activité (\%) \\
\hline 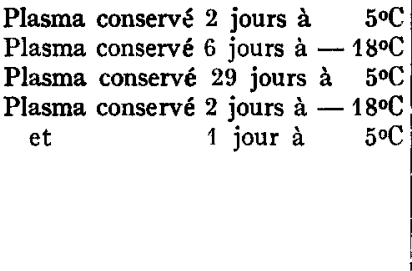 & $\begin{array}{r}100 \\
98 \\
97 \\
103\end{array}$ & $\begin{array}{l}\text { Foie conservé congelé } 2 \text { jours } \ldots \\
\text { Foie conservé congelé } 5 \text { jours } \ldots \\
\text { Foie conservé congelé } 12 \text { jours } \ldots \\
\text { Foie congelé } 2 \text { jours et conservé } \\
\text { à l'état d'homogénat : } \\
24 \text { heures à } 5^{\circ} \mathrm{C} \ldots \ldots \ldots \\
24 \text { heures à }-18^{\circ} \mathrm{C} \ldots \ldots \ldots \\
9 \text { jours à } 5^{\circ} \mathrm{C} \ldots \ldots \ldots \\
9 \text { jours à }-18^{\circ} \mathrm{C} \ldots \ldots \ldots\end{array}$ & $\begin{array}{r}103 \\
98 \\
51 \\
63\end{array}$ \\
\hline
\end{tabular}

\section{3. Étude de la lacticodéshydrogénase plasmatique}

Les résultats d'ensemble concernant l'activité enzymatique des différents lots sont représentés sur la figure 2 a où sont portées en abscisse les dates de prélèvement et en ordonnées les activités correspondantes. On peut faire les observations suivantes :

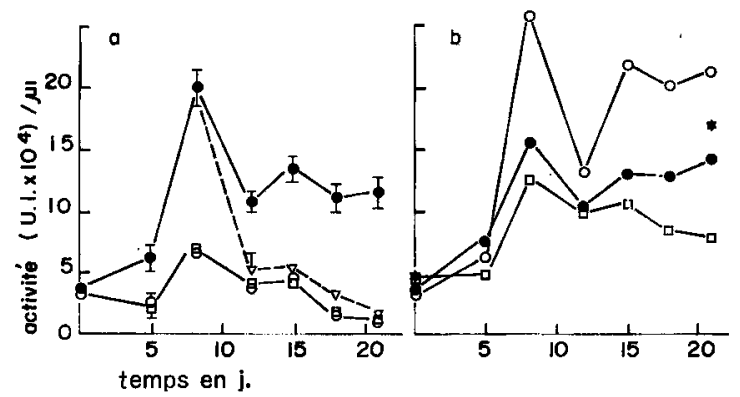

FIG. 2. - Evolution de l'activité de la S-LDH au cours du gavage

Les activités enzymatique sont exprimés en Unités Internationales par $\mu l$ de plasma $2 a(0)$ et ( $\square$ ), témoins $(\bullet)$ : gavés $(\nabla)$ : " dégavés », $2 b(\bullet)$ activité moyenne des animaux gavés après déduction des variations du lot témoin (o), cas d'un animal présentant une forte stéatose (foie de $93 \circ \mathrm{g}$ ) (口), cas d'un animal présentant une stéatose plus faible (foie de $490 \mathrm{~g}$ ) $\left(^{*}\right)$, résultats d'une autre expérience (SzYLIT, LECLERQ, IVOREC-SZYLIT, I 968). 
a) Animaux non gavés.

On ne note aucune différence entre l'activité des lots A et B. Il s'ensuit que le prégavage ainsi que la nature du régime sont sans effet sur la concentration de la S-LDH des oiseaux non gavés.

b) Animaux gavés.

L'activité S-LDH des oies gavées est toujours supérieure à celle des oies témoins. Des différences importantes apparaissent dès le $5^{\mathrm{e}}$ jour, s'amplifient et sont maximales 8 jours après le début du gavage. Selon les sujets, l'activité est 2 à 4 fois celle des témoins. Dans nos conditions de prélèvement, le $8^{\mathrm{e}}$ jour marque la fin d'une première étape dans 1'évolution de la $\mathrm{S}-\mathrm{L} \mathrm{DH}$. La seconde étape est caractérisée par une baisse d'activité plus ou moins importante suivant les animaux. On observe cette évolution pour tous les oiseaux quel que soit le résultat final de la stéatose. Au cours de la phase finale, l'activité augmente à nouveau chez certains sujets (oie 930), ou reste stationnaire ou encore diminue légèrement (oie 490 ) (fig. $2 b$ ).

Les oies dont la concentration de la S-LDH croît pendant la $3^{e}$ phase sont celles dont le plasma possède la plus grande activité enzymatiqua après une semaine de gavage. Ces variations individuelles ne sont pas aléatoires contrairement à ce que l'on observe dans le cas des témoins, et leur signification en relation avec l'importance de la stéatose au $2 \mathrm{I}^{\mathrm{e}}$ jour sera discutée plus loin.

c) Animaux " dégavés ".

L'activité enzymatique tend à revenir à son niveau normal dès que le gavage est interrompu et ceci d'autant plus rapidement que l'interruption est plus précoce (fig. $2 a$ et 3). L'accroissement de l'activité est presque totalement réversible si l'in-

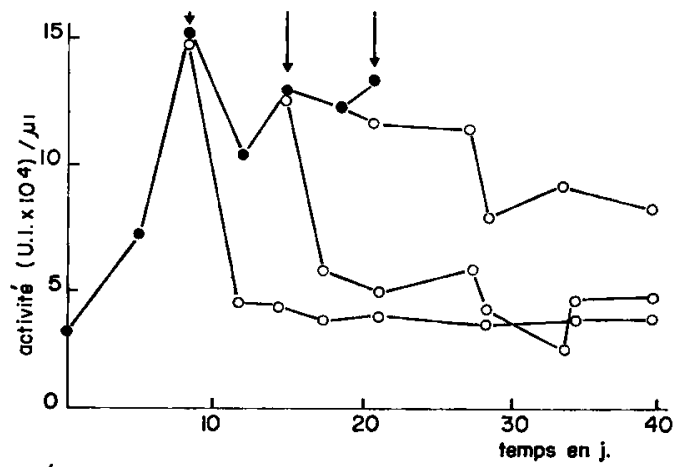

FIG. 3. - Evolution de l'activité de la $S$-LDH apres interruption du gavage d différentes périodes indiquées par les fleches

Les activités enzymatiques sont exprimées en unités Internationales par $\mu l$ de plasma $(\bullet)$, animaux gavés (o), animaux dégavés

terruption du gavage a lieu pendant les $I_{5}$ premiers jours. Par contre, au bout de la $3^{e}$ semaine, la réversibilité n'est plus totale et il faut attendre 20 jours pour que 1'activité soit réduite de moitié. Ceci suggère que l'accroissement de la S-L,DH résulte de mécanismes différents en fin et en début de gavage.

d) La constante de MICHAELIS relative au pyruvate mesurée entre I, 6 et $324 \cdot \mathrm{IO}^{-5} \mathrm{M}$ est égale à $\mathrm{I}, 3 \pm 0,10.10^{-4} \mathrm{M}$ à $\mathrm{pH} 7,6$ et $25^{\circ} \mathrm{C}$ pour des concentrations en $\mathrm{NADH}$ comprises entre $\mathrm{I}, 07$ et $3,2 \mathrm{I}$. $\mathrm{IO}^{-4} \mathrm{M}$. Cette valeur reste inchangée au cours du gavage. 


\section{4. Étude de la lacticodéshydrogénase du foie}

a) Animaux gavés.

Les différences d'activité mesurées à différents niveaux du petit lobe ne sont pas significatives, aussi, l'activité totale du foie est calculée en supposant une distribution uniforme de l'enzyme. On prend comme terme de comparaison la valeur mesurée au $2 \mathrm{I}^{\mathrm{e}}$ jour dans le cas des animaux témoins $(34000 \pm 2000 \mathrm{UI})$. L'activité par gramme de protéine $\left(2,2 \cdot 10^{-3} \mathrm{UI}\right)$ est du même ordre que celle mesurée sur d'autres espèces : I,6. Io $^{-3}$ UI et $2,6$. IO $^{-3}$ UI respectivement chez le veau (GUTTI,ER et CLAUSEN, I967) et le rat (Schmidt et al., I966). Les variations de la F-LDH en fonction du temps sont représentées sur la figure $4 a$. Sur ce même graphique, nous portons les résultats obtenus au cours d'une expérience précédente (SzYLIT, LECLERCQ et IVORECSzyıIT, I968). Le gavage est caractérisé par une synthèse importante de cet enzyme pendant les 5 premiers jours, puis l'activité enzymatique cesse d'augmenter alors que la stéatose évolue toujours (fig. 4 b).

b) Animaux "dégavés".

Après interruption du gavage, le taux de la F-LDH revient à sa valeur normale, et très rapidement il devient difficile d'établir une distinction entre animaux témoins et "dégavés" (fig. 4 a). Cependant, il n'en est pas de même en ce qui concerne le poids du foie (fig. 4 b) et celui de l'animal (fig. $4 c$ ). Après interruption du gavage au $2 \mathrm{I}$ jour, la réversibilité partielle dans le cas de l'enzyme plasmatique, est totale dans celui de la F-LDH. Si, comme nous le pensons (Szylit et IvoreC-Szyi,IT, I967; SzYLIT,
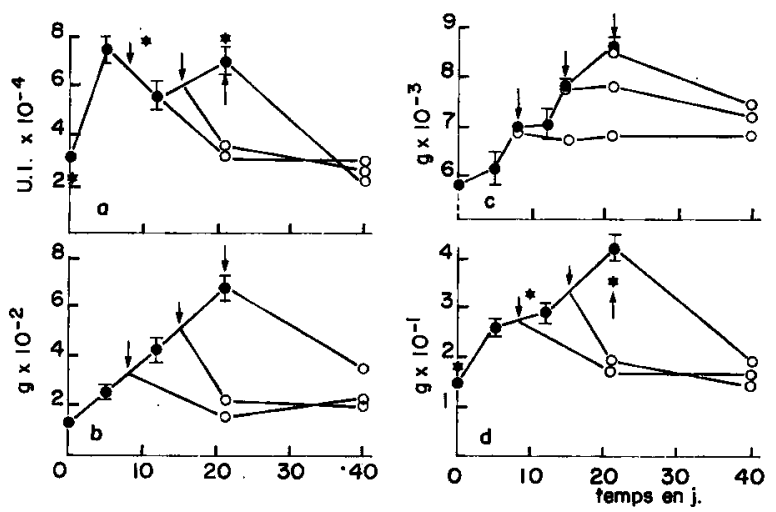

FIG. 4. - Evolution de la quantité totale de F-LDH (3a) du poids du foie $(3 b)$ du poids corporel $(3 c)$ et de la quantité totale des protéines solubles du foie ( $3 d)$ au cours ( $\bullet$ ) et apres interruption (o) du gavage $(*)$,

autre expérience (SzYLIT, LECLERCQ, IvOREC-SzyLIT I968). L'activité de la F-L'DH est exprimée en unités internationales. On prend comme valeur au temps o les résultats obtenus au $2 I^{e}$ jour dans le cas des animaux témoins.

LeCLERCQ et IVOREC-SzYIIT, I968), l'accroissement de la S-LDH est d'origine hépatique, cela reviendrait à supposer que dans certaines conditions, l'activité synthétique du foie revient à son niveau normal alors que les mécanismes responsables de la libération de la F-I,DH dans la circulation générale restent perturbés. 
c) Les variations de l'activité rapportées au gramme de foie frais sont portées sur la figure $5 a$.
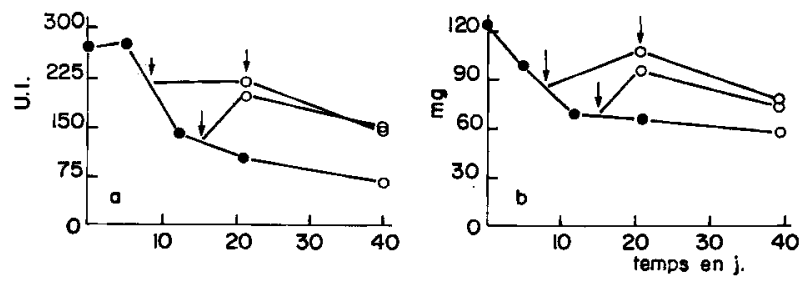

Fig. 5. - Variations de la F-LDH $(5 a)$ et des protéines solubles du foie ( $5 b)$ rapportées a I gramme de foie frais au cours (๑) et apres interruplion (o) du gavage

d) La constante de MichaEı,Is relative au pyruvate est égale à $\mathrm{I}, \mathrm{I} 5 \pm 0, \mathrm{I}_{3} \cdot \mathrm{IO}^{-4} \mathrm{M}$ à $\mathrm{pH} 7,6$ et $25^{\circ} \mathrm{C}$.

\section{Analyse électrophorétique de la $L D H$ du plasma et du foie}

Dans la figure 6, nous montrons des échantillons de plasmas et d'homozénats de foie correspondant à des oies témoins et gavées. L'analyse électrophorétique confirme les résultats obtenus par le dosage spectrophométrique : la concentration des lacticodéshydrogénases est supérieure chez l'animal gavé. Ces enzymes ne sont pas homogènes : on distingue plusieurs bandes régulièrement espacées entre elles, que l'on désigne par $\mathrm{LDH}_{5}, \mathrm{LDH}_{4} \ldots \mathrm{LDH}_{1}$ suivant la terminologie de WIELAND et PFEIDERER (I957). $\mathrm{LDH}_{5}$ est la fraction la plus lente.

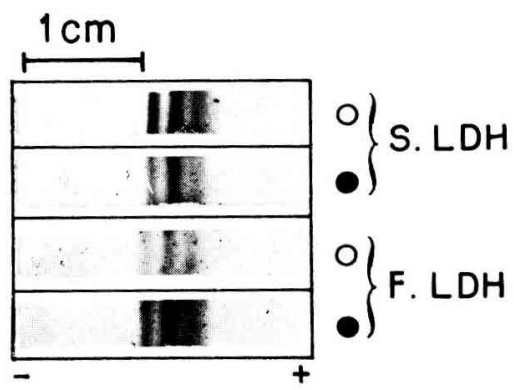

FIG. 6. - Analyse électrophorétique des lacticodéshydrogénases du plasma et du foie Animaux gavés (•). Témoins (०)

L'enzyme du plasma de 1'oie non gavée renferme essentiellement les isoenzymes 5 , 4 et 3. L'importance relative de la fraction 5 par rapport à la fraction 4 est variable suivant l'animal ; ces 2 isoenzymes représentent $90 \mathrm{p}$. roo de 1'activité totale. A la suite du gavage, la concentration de chaque fraction est accrue fortement et les 2 bandes mineures $\mathrm{LDH}_{5}$ et $\mathrm{LDH}_{5}$ apparaissent plus nettement. Ces dernières sont difficiles à mettre en évidence sur le cliché photographique, par suite de leur dilution. Enfin, on observe un phénomène nouveau d'ordre qualitatif : la concentration de la fraction 4 devient supérieure, de façon systématique à celle de la fraction 5 . 
L'enzyme extrait du foie renferme les mêmes isoenzymes 3, 4 et 5 mais la fraction 4 est toujours prédominante. A la suite du gavage, on observe un accroissement uniforme de la concentration de ces bandes et les fractions $I$ et 2 deviennent visibles.

\section{Evolution des protéines du plasma et du foie}

Le gavage est caractérisé par un faible accroissement non significatif de la protéinémie (fig. 7). L'analyse par ultracentrifugation du sérum de l'oie normale montre l'existence de 3 constituants $A, G$ et $M$. Les valeurs des coefficients de sédimentation à $20^{\circ} \mathrm{C}$ extrapolées pour une concentration nulle sont respectivement 4,8-6,5 et I8. L'albumine représente $85 \mathrm{p}$. Ioo du mélange et les macroconstituants $5 \mathrm{p}$. Ioo environ (sérum dilué 6 fois). Nous n'avons pas constaté de différence entre les plasmas des animaux témoins et gavés.

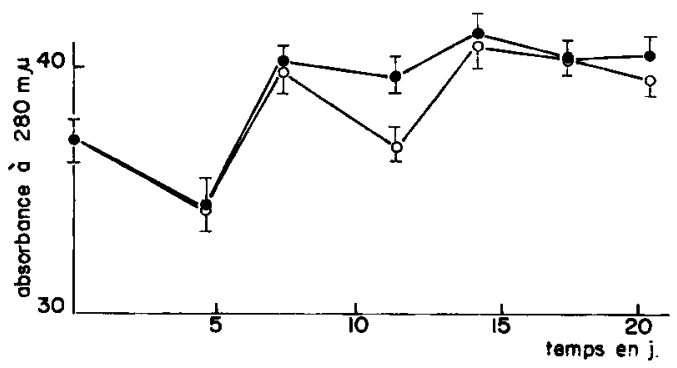

Fig. 7. - Évolution de la protéinimie au cours du gavage pour les animaux témoins (०) et gavés (•)

On mesure l'absorption à $280 \mathrm{~m} \mu$ sous I $\mathrm{cm}$ d'épaisseur

L'évolution des protéines hydrosolubles du foie pour les lots $A, B$ et $C$ est indiquée dans la figure $4 d$ sur laquelle on a aussi représenté les résultats correspondant à une autre expérience (SzYLIT, I,ECLERCQ et IVOREC-SZyLIT, I968). Cette fraction protéique s'accroît régulièrement au cours du gavage, mais par suite de l'accumulation importante de lipides, le rapport protéines hydrosolubles/poids frais diminue. Rapportée au gramme de foie frais, la vitesse de synthèse des protéines (fig. $5 b$ ) est inférieure à celle de la F-LDH. L'évolution de la LDH dont la concentration cesse d'augmenter à partir du $5^{\mathrm{e}}$ jour est donc différente de celle de la fraction protéique. Quand le gavage est interrompu, le taux des protéines hydrosolubles redevient normal, suivant en cela l'évolution de la F-L,DH. L'électrophorèse permet de distinguer ${ }_{5} 5$ à 20 zones protéiques : il ne nous a pas été possible d'établir des différences significatives entre les homogénats de foie des oies normales et gavées. D'après l'ultracentrifugation analytique, cette fraction est un mélange complexe formé d'au moins 4 constituants.

Nous avons montré (SzYLIT, LECLERCQ, IVOREC-SzYLIT, I968) que la stéatose s'accompagne d'une augmentation considérable (multiplication par ro) de la teneur en acide lactique dans le foie. On note aussi un accroissement de la teneur en ARN soluble, plus important proportionnellement que celui de la fraction protéique (SzYLIT, non publié). 


\section{DISCUSSION}

\section{Remarques concernant l'évolution de la S-LDH des oies témoins}

Les variations de concentration de la $\mathrm{S}-\mathrm{L}, \mathrm{DH}$ présentent un double aspect car certains phénomènes évolutifs concernent non seulement tous les oiseaux gavés du lot $\mathrm{C}$ mais aussi tous les oiseaux témoins des lots A et B. Ainsi, la S-LDH des oies témoins évolue de façon parfois importante alors qu'apparemment, les conditions de l'expérience sont inchangées. De plus, ces variations sont aussi visibles sur la courbe de protéinémie (fig. 7). Ces fluctuations sont donc la conséquence d'un facteur commun à tous les animaux et reflètent des perturbations physiologiques d'ordre général. Il est difficile de se prononcer sur l'origine de ces fluctuations ; WROBLEWSKI (I96I) en observe de similaires chez le lapin.

Certaines causes de perturbations sont incontrôlables :

choc physiologique consécutif au transport, au changement d'habitat, aux diverses manipulations quotidiennes, etc.

Comme l'expérience s'est déroulée en hiver dans un bâtiment non chauffé, nous avons pensé à un effet possible de la température ambiante. Dans la figure $I$, nous comparons l'évolution de la S-LDH (fig. I $d$ ) aux variations de la température moyenne quotidienne (fig. I $c$ ) et à celles de la consommation d'aliments (fig. I $a$ ). Nous constatons que les variations aléatoires de la consommation des oies témoins ne peuvent rendre compte du phénomène observé. On remarque par contre, sans qu'i1 soit permis de conclure, que le maximum au $8^{\mathrm{e}}$ jour et le minimum au $\mathrm{I} 2^{\mathrm{e}}$ jour de la courbe relative à 1'enzyme suivent de 24 heures un maximum et un minimum très prononcé de la courbe de température. Dans cet intervalle de temps (du $8^{\mathrm{e}}$ au $\mathrm{I}^{\mathrm{e}}$ jour) l'accroissement du poids des oiseaux est ralenti (fig. I $b$ ).

\section{Relations entre S-LDH et F-LDH au cours du gavage}

A ces fluctuations s'ajoutent les variations propres aux animaux du lot $\mathrm{C}$, donc consécutives au gavage.

On n'attribue pas de rôle métabolique à la lactico-déshydrogénase du plasma, considérée comme un débris cellulaire et dont la concentration est inférieure à celle des enzymes tissulaires. Dans des conditions physiologiques normales, la concentration en S-LDH, relativement stable, traduit un équilibre entre 1'apport (diffusion et lyse cellulaire) et le catabolisme de cette enzyme. Nous montrons que cet état d'équilibre est altéré quantitativement et qualitativement à la suite du gavage. D'une part, la concentration en $\mathrm{S}-\mathrm{LDH}$ augmente de façon très nette : un microlitre de plasma réduit 4. $10^{-10}$ moles de pyruvate par minute en début de gavage et $\mathrm{I} 6 . \mathrm{ro}^{-10} \mathrm{en}$ fin de gavage. Le premier chiffre est du même ordre que celui rapporté par ZIMMERMaN (I965) : 
r,2. IO $^{-10}$. D'autre part, l'importance relative des isoenzymes est modifiée : la proportion de la forme 4 est accrue.

L'excès de LDH observé dans le plasma provient sans conteste d'organes profondément affectés par le gavage. La stéatose hépatique en est la manifestation la plus importante : la figure 8 montre qu'il n'y a aucune commune mesure entre l'accroissement du poids du foie et celui du poids vif de l'animal ou de celui d'un autre organe tel que le pancréas. On peut donc penser que l'enzyme plasmatique excédentaire provient essentiellement du foie. Cette hypothèse se déduit entre autre de l'analyse électrophorétique comparative de la $L_{L} D H$ du foie et du plasma qui montre qu'au cours du gavage, les diagrammes électrophorétiques deviennent très ressemblants (fig. $6 a$ ).

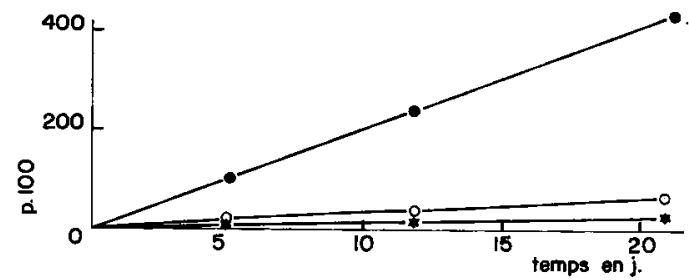

Fig. 8. - Accroissement relatif du poids du foie (•); du pancréas $\left(^{*}\right)$; et du poids corporel (O), au cours du gavage

D'autres faits sont en faveur de cette hypothèse. Nous observons une corrélation entre l'importance de la stéatose et la concentration en S-LDH au moins à 2 étapes du gavage, au $8^{\mathrm{e}}$ et au $2 \mathrm{I}^{\circ}$ jour. La figure $9 a$ montre que cette concentration est
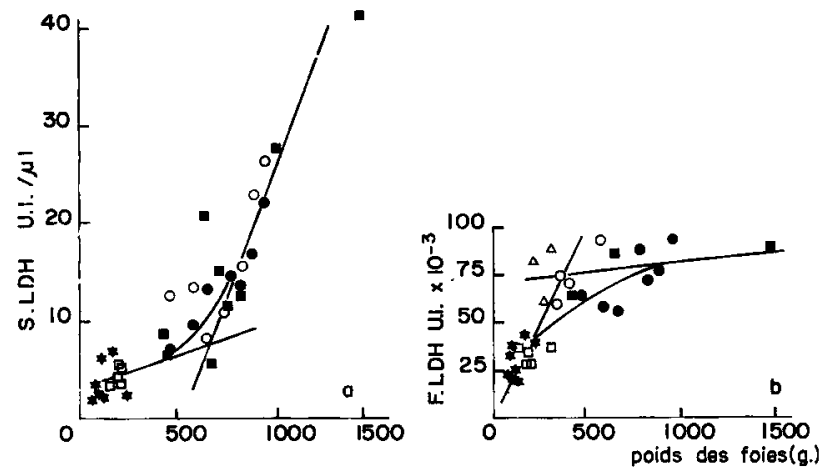

FIG. 9. - Activilés $S-L D H$ (9 a) et F-LDH (9 b) en fonction du pcids du fcie

$\left(^{*}\right)$ témcins; $(\square)$ dégavés au $7^{\mathrm{e}}$ et $14^{\mathrm{e}}$ jour mesurés au 21 e jour;
$(0)$ gavés 8 jours; $(\bullet)$ gavés $2 \mathrm{I}$ jours. Autre expérience $(\square)$, gavés $2 \mathrm{I}$ jours; $(\square)$, mi-gavés

d'autant plus élevée que le poids du foie est plus important. C'est d'ailleurs chez les oies les mieux gavées que le taux de la S-LDH croît au cours de la dernière semaine alors qu'il reste constant pour les autres oiseaux (fig. 2 b).

L'exemple d'un animal qui a présenté une réactivité anormale est particulièrement suggestif. La stéatose était considérablement développée à mi-gavage, et à une 
activité F-LDH exceptionnelle correspondait une activité S-LDH très importante (tabl. 2).

\section{TABLEAU 2}

Valeurs moyennes au $11^{\mathrm{e}}$ et $21^{\mathrm{e}}$ jour de la $S-L D H$, de la $F-L D H$ et du poids du foie comparées d̀ celles d'un animal présentant une réponse exceptionnelle au gavage

\begin{tabular}{|c|c|c|c|}
\hline & $\begin{array}{l}\text { Valeur moyenne } \\
\text { au } 11^{\circ} \text { jour }\end{array}$ & $\begin{array}{l}\text { Valeur moyenne } \\
\text { au } 21^{e} \text { jour }\end{array}$ & $\begin{array}{c}\text { Valeur Animal } 750 \\
11^{\mathrm{e}} \text { jour }\end{array}$ \\
\hline F-LDH & $60.10^{3}$ UI & $70.10^{3} \mathrm{UI}$ & $159.10^{3} \mathrm{UI}$ \\
\hline S-LDHi $\ldots \ldots$ & $11,5.10^{-4} \mathrm{UI}$ & $16.10^{-1} \mathrm{UI}$ & $95.10^{-4} \mathrm{UI}$ \\
\hline Poids du foie.. & $400 \mathrm{~g}$ & $700 \mathrm{~g}$ & $750 \mathrm{~g}$ \\
\hline
\end{tabular}

\section{Mécanismes possibles de la libération de F-LDH dans la circulation}

Pendant les 5 premiers jours du gavage, le foie est le lieu d'une très grande activitê synthétique qui porte sur les protéines solubles, l'ARN soluble (SzYLIT, non publié) et la LDH. Il s'ensuit un renforcement du gradient de concentration entre le milieu intracellulaire et le sang circulant, ce qui provoque une augmentation de la vitesse de diffusion de la F-LDH de l'hépatocyte vers la périphérie. La localisation presqu'exclusive de cet enzyme dans le compartiment cytoplasmique (Schmid et al., r966) ne peut que faciliter ce mouvement.

Cette augmentation de la vitesse de diffusion doit s'accompagner à partir du.

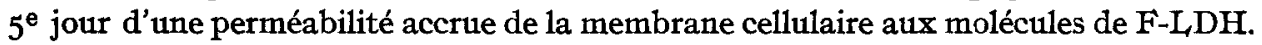
En effet, la concentration en S-LDH croît brutalement sans qu'on puisse observer de. lésions cellulaires à ce stade (WYRSYKowSKI et BIELINSKY, I967 ; LABIE, I967) alors. que l'activité F-LDH cesse d'augmenter.

Pendant la deuxième semaine de l'expérience, ces deux enzymes évoluent plus. faiblement (fig. 3 et $4 a$ ) traduisant peut-être l'établissement d'un nouvel état d'équilibre après le choc dû à l'instauration du gavage. De plus, la quantité d'enzyme qui quitte le foie ne peut diminuer de façon notable le taux de F-LDH. En effet, 1'activité F-L,DH totale est près de 300 fois supérieure à celle de la $\mathrm{S}-\mathrm{LDH}$ si on suppose que le plasma représente $6 \mathrm{p}$. Ioo du poids vif de l'animal. Comme à la fin de la $\mathrm{I}^{\mathrm{r} \theta}$ semaine, 1'accroissement du taux de la S-LDH est aussi totalement reversible en fin. de $2^{\mathbf{e}}$ semaine.

Mais au cours de la $3^{\mathbf{e}}$ semaine, apparaissent des phénomènes nouveatux qui semblent indiquer une modification des mécanismes responsables de la libération de la $L D H$. On a vu que la S-L,DH croît à nouveau chez certaines oies; ainsi, en fin de gavage, les activités S-LDH des oies dont le foie est inférieur et supérieur à $600 \mathrm{~g}$ sont dans le rapport I,4-4. Donc à ce stade, l'activité de la S-L,DH dépend fortement de l'importance de la stéatose, contrairement à ce que l'on observe pour l'activité de la F-LDH (fig. 9 b). Dans ces conditions, nous ne pensons pas qu'une simple augmentation de perméabilité cellulaire puisse rendre compte à elle seule de ces différences mais qu'elle doit s'accompagner d'une rupture de la membrane de certaines cellules comme 
le laisserait supposer 1'augmentation des transminases sériques (FORENBACHER, I967); c'est justement dans des foies très lourds, présentant une surcharge graisseuse importante du type macro-vacuolaire que l'éclatement de la membrane cellulaire a pu être observé (LABIE, I967). Les altérations du foie sont à leur maximum après une $4^{\mathrm{e}}$ semaine de gavage ; à côté d'une grave dégénérescence parenchymateuse, il apparaît des symptômes de nécrobiose et même un commencement de processus cirrhogène (FORENBACHER, I967). Les résultats des expériences d'interruption de gavage au $2 \mathrm{I}^{\mathrm{e}}$ jour sont en accord avec cette hypothèse. L'activité $\mathrm{LDH}$ du foie redevient normale bien que le poids de 1'organe reste élevé (fig. $3 b$ ) et sa structure spongieuse. Par contre, le taux de la S-LDH ne diminue que lentement et reste à un niveau d'autant plus élevé que le foie est plus lourd. D'ailleurs, 3 semaines après l'interruption, ce taux est encore le double de celui d'une oie non gavée (fig. 2). L'activité $\mathrm{S}-\mathrm{LDH}$ résiduelle, après interruption du gavage, serait donc une mesure de l'importance de l'altération cellulaire. Des observations du même ordre faites chez le rat montrent une augmentation de la vitesse de libération de certaines enzymes au cours de l'étape nécrotique qui suit la dégénérescence graisseuse induite par la thioacétamide (WYRZYKoWSKI et BIELINSKI, I967).

\section{Interprétation de ces phénomènes}

Nous avons vu que l'évolution de la F-LDH n'est pas un phénomène isolé. WYRzYKOWSKI (I967), a d'ailleurs observé une augmentation du nombre des cellules, Cette intense activité anabolique peut être la conséquence d'un dérèglement hormonal comme le suggèrent les travaux de WEBER (I963), de GoODFRIEND (I964) et de BARgoni (I96I) qui montrent que la cortisone, l'œstradiol et les hormones thyroidiennes favorisent la synthèse de la F-LDH, particulièrement des formes 5 et 4 .

Il est intéressant de connaître plus précisément le mécanisme de cette fuite extracellulaire et le moment où elle ne peut plus être considérée comme une réaction d'adaptation physiologique. A côté de cas extrêmes pour lesquels la nécrose cellulaire est un fait bien établi, certains exemples montrent que cette fuite transmembranaire apparaît, en absence de tout changement histologique apparent de l'hépatocyte (ScHmidT et al., I966 ; HENLEY et al., I959).

Le mécanisme de la perméabilité cellulaire est encore inconnu en ce qui concerne le mouvement des grosses molécules comme la $\mathrm{LDH}$ (poids moléculaire voisin de I30 000). La distension de la membrane provoquée par les inclusions lipidiques pourrait à elle seule expliquer l'accroissement réversible de la perméabilité, soit par une augmentation de la surface d'échange, soit par une dilatation de "pores " hypothétiques. Les modifications de la perméabilité cellulaire peuvent être induites par des changements de la polarisation nembtahaire créés par une perturbation du métabolisme hydrominéral (DELAHAYES, DE LEIRIS et CORABOEUF, I965) ou encore par certains effecteurs tels que les corticostéroìdes (JACEY et SCHAEFER, I967).

Le problème des lésions cellulaires liées au développement de la stéatose hépatique chez l'oie reste très controversé. Tous les auteurs s'accordent pour décrire un accroissement de la taille de l'hépatocyte par suite d'une surcharge lipidique qui réduit le cytoplasme à une mince pellicule périphérique et à quelques filets intervacuolaires. Certains seulement décrivent en fin de gavage l'apparition de lésions discrètes du noyau et de la membrane (WYRZYKowsky et BIFLINSKI, I967; MAHX et 
Rowson, I965). Nous avons vu qu'il suffit d'un très petit nombre d'hépatocytes lésés pour que l'activité de la S-LDH s'accroisse de façon considérable et c'est sans doute pour cette raison que les lésions sont difficilement détectables par les méthodes histologiques usuelles.

Quelle que soit l'espèce considérée, l'interprétation des changements de la LDH rend compte bien plus de la nature de l'organe "lésé " que de la nature de l'agent responsable de cet état pathologique. Ainsi, l'irradiation gamma (HAWRYLEWICZ et BLAIR, Ig66), l'administration d'agents toxiques tel que le tétrachlorure de carbone (WIEME et DEMEUlENAERE, I959), certaines formes de cancer (BoDANSKI et ScHOLIER, I956) produisent qualitativement les mêmes effets que le gavage au niveau de la S-LDH. L'accroissement de la perméabilité cellulaire de l'hépatocyte mesuré par l'élévation du taux de la LDH plasmatique, semble être la première réponse au gavage bien avant l'apparition de toutes modifications histologiques.

Rę̧u pour publication en janvier 1969.

\author{
SUMMARY
}

STUDY OF LACTIC DEHYDROGENASE IN PLASMA AND LIVER

IN GEESE DURING STEATOSIS CAUSED BY CRAMMING

\begin{abstract}
Lactic dehydrogenases were studied in liver (F-LDH) and plasma (S-LDH) in geese during steatosis produced by cramming for 3 weeks continuously or after interruption at different stages.

During the first 5 days of cramming there was extensive synthesis of F-LDH (fig. 4) which concerned equally the isoenzymes 4,5 and 3 ; fraction 4 was the greatest (fig. 6).

Concentration of S-LDH increased during cramming (fig. 2). Three phases were seen in this development : rapid increase from the fifth day and maximum concentration at the eighth ; a small decrease during the second week ; stabilization or another small increase during the third week. Concentrations on the eighth and twenty-first days were greater when livers were heavier at 2 I days (fig. 9A). The increase was essentially in isoenzyme 4 so that after cramming the electrophoretic diagrams became very similar. The qualitative and quantitative changes in S-LDH can be interpreted as resulting from liberation of F-LDH into the general blood circulation.

If cramming was stopped during the first 15 days F-LDH and S-LDH activities rapidly returned to their normal values. On the other hand, at the end of cramming only F-LDH returned to normal (fig. 4) and S-LDH continued to depend on the degree of stetatosis (fig. 3 ).

The results suggest a change in the mechanism of release of F-LDH : following a reversible increase in membrane permeability, at the end of cramming there may possibly be cellular lesions. The causes of this intense metabolic activity and the mechanisms responsible for the movement of F-LDH are discussed. Increase in permeability of the liver cells, measured by the increase in S-LDH, seems to be the first response to cramming, even before the appearance of any histological modifications. At the end of cramming activity of S-LDH would be a measure of changes in cells.
\end{abstract}

\title{
RÉFÉRENCES BIBLIOGRAPHIQUES
}

Bargoni N., 196r. Uber die Leberglycolyse von mit Schildrüse gefütterten Ratten. Hoppe Seyler's Z. Physiol. chem. 126, 65-72.

Bodansky O., Scholler J., I956. Comparaison of phosphohexose isomerase and lactic-dehydrogenase activities in plasma, liver, and tumor tissue of tumor bearings rats. Cancer, Res., 16, 894-897.

Chrambach A., Retsfeld R. A., Wyckoff M., Zaccari J., r967. A procedure for rapid and sensitive staining of protein fractionated by polyacrylamid gel electrophoresis. Anal. Biochem., 20, 150-154.

Annales de Biologie animale. - I969. 
Delahayes J., De Leiris J., Coraboevf T., 1965. Liberation de lacticodeshydrogenase (LDH) par le cour de rat sous l'action de milieux hyperpotassiques. C. R. Acad. Sci. Paris., 261, 5222-5225.

Dewey M., ConkLIN J., I960. Starch gel electrophoresis of lacticodehydrogenase from rat kidney. Proc. Soc. Exp. Biol. Med., 105, $492-497$.

Forenbacher S., r967. Changements dans la composition du foie et du sang des oies au cours du gavage. Dans Journées d'étude sur l'élevage et la production de l'Oie. I. N. R. A., éd., 22-27 mai, i 2 pages.

GoODFRIEND T. L., KaPLAN N. O., 1964. Effect of hormone administration on lactic dehydrogenase. $J$. Biol. Chem., 239, I30-135.

Guttler F., Clausen J., 1967. Cellular compartmentalisation of lactate dehydrogenase isoenzymes. Enzym. Biol. Clin., 8, 456-470.

HAWrylewicz E. J., BLAiR W. H., 1966. Effect of gamma and proton irradiation on lacticodehydrogenases isoenzymes. Radiation. Res., 28, 538-547.

Henley K. S., Wiggins H.S., Pollard H. M., Dallaert E., I959. The transaminase content of parachymatous liver cells. Gastr. entérologie., 36, I-6.

Ivorec-Szylit O., Szylit M., r965. Contribution à l'étude de la dégradation des glucides dans le jabot du coq. I. Mise en évidence et dosage des stéréisomères $\mathrm{D}$ et $\mathrm{L}$ lactate. Ann. Biol. anim. Bioch. Biophys., 5, 353-360.

JACEY M. J., Schaefer K. E., 1967. Regulation of plasma lactic dehydrogenase on chronic respiratory acidosis. Amer. J. Physiol., 212, 859-863.

Kubowitz F., OTt P., 1943- Isolierung und Kristallisation eine gerung Ferment aus Tumoren. Biochem Z., 314, 94-II7.

LABIE C., 1967. Évolution du foie de l'Oie au cours du gavage. Journées d'étude sur l'élevage et la production de l'Oie, I. N. R. A., éd., 22-27 mai, 3 pages.

MaHY W. B., Rowson K. E. K., 1965. Isoenzymic specificity of impaired clearance in mice infected with Riley virus. Science, 149, 756-757.

Markert C. L., Moller F., 1959. Multiple forms of enzymes tissue, ontogenic and species specific patterns. Proc. Nat. Acad. Sci., U.S. A., 45, 753-763.

Pokrovski A. A., Archakov A. I., Guerassimov, A. M., Dervichenskit W. M., Lasheneva N. V., r967. Sorbitol dehydrogenase, alanine aminotransaminase and Phosphohexose isomerase in blood plasma of rat with acute toxic liver degeneration. Vop. Med. Khim., 13, 511-5I6.

Schmidt E., Schmidt F. A., Herfarth Opitz K., Vogell W., ig66. Studien Zum Austritt von ZellEnzymznam Modell der isolierun, Perfundirecten Ratten leber (III mitteilung). Biochem. Z., 329, $112-116$.

Shonk C. E., Boxer G. E., 1964. Enzyme patterns in human tissues. I. Methods for the determination of glycolitic enzymes. Cancer, 24, 709-7II.

Szylit M., Ivorec-Szylit O., I967. Hétérogénéité et propriétés de la LDH du sérum de l'Oie. $C$. $R$. Acad. Sci., Paris, 285, I84I-I844.

SzylıT M., I968. Électrophorèse verticale en gel d'Acrylamide. Dispositif simplifié. Path. Biol., 16, $247^{-256}$.

Szylit M., Leclerce B., Ivorec-Ssylit O., I968. Relations spécifiques entre les lacticodeshydrogénases du sérum et du foie chez l'Oie : détermination quantitative et caractérisation électrophorétique. $C$. $R$. Acad. Sci. Paris, 266, 952-955.

Warburg O., Christian W., I94I. Isolierung-und Kristallisation des gärungsferments Enolase. Biochem. Z., 310, 384-42I.

WEBER G., I963. Actinomycine inhibition of cortisone induced synthesis of hepatic gluconeogenis enzyme. Science, 142, 390-39r.

Wieland T., Pfleifeder G., 1957. Nachweis der Heterogenitaät von Milchsaüre dehydrogenasen verschiedenen Ürspring durch Trägen electrophoresis. Biochem. Z., 329, I I 2-I I6.

Wieme R. J., Demeulenaere I., 1959. Enzyme-electrophoresis of the lactic acid dehydrogenase selective test of parenchymatous intregrity. Acta Gasiroenterol. Belg., 29, 69-79.

WroblewSKi F., Gregory K. F., r96r. Lactic dehydrogenases isoenzymes and their distribution in normal tissue and plasma and in disease states. Ann. N. Y., Acad. Sci. U. S. A., 45, 753-763.

Wyrzykowski Z., Bielinski K., r967. Microscopic picture of goose liver during forced feeding. Journées d'étude sur l'élevage et la production de l'OIe. I. N. R. A., éd. 22-27 mai, 2 pages.

Zimmerman H. J., Schwartz M. A., Boley L. E., West M. J., 1965. Comparative serum enzymology. Lab. and Clin. Med., 66, 961-972. 